\title{
The HEPD apparatus for the CSES mission
}

\author{
Beatrice Panico* \\ INFN Napoli, I-80126 Naples, Italy \\ E-mail: bpanicoena.infn. it \\ G. Osteria, F. Perfetto, V. Scotti
}

INFN Napoli, I-80126 Naples, Italy

P. Cipollone, C. De Donato, C. De Santis, L. Marcelli, G. Masciantonio, M. Mergè, F. Palma, P. Picozza, A. Sotgiu, R. Sparvoli

INFN, Sezione di Roma Tor Vergata, I-00173 Rome, Italy

\author{
A. Contin, C. Guadalini, G. Laurenti, M. Lolli, F. Palmonari, L. Patrizii, M. Pozzato \\ INFN, Sezione di Bologna, I-40127 Bologna, Italy
}

\section{Ricci, B. Spataro}

INFN, Frascati National Laboratories, I-00044 Frascati - RM, Italy

\author{
W.J. Burger, R. Battiston, F.M. Follega, R. luppa, I. Lazzizzera, C. Manea, I. \\ Rashevskaya, E. Ricci, V. Vitale, \\ INFN, Sezione di Trento, I-38123 Povo - TN, Italy
}

G. Ambrosi, M. Ionica, M. Sposito

INFN Perugia, I-06123 Perugia, Italy

\section{S. Bartocci, L. Conti}

International Telematic University UNINETTUNO, I-00186 Roma, Italy

G. Castellini, L. Pacini, S.B. Ricciarini

IFAC-CNR, I-50019 Sesto Fiorentino - FI, Italy

\begin{abstract}
The CSES (China Seismo-Electromagnetic Satellite) mission will investigate the structure and the dynamic of the topside ionosphere, will monitor electric and magnetic field and high energy particle fluctuations, searching for their correlations with the geophysical activity, in order to contribute to the monitoring of earthquakes from space. The High-Energy Particle Detector (HEPD) is one of the payloads of the CSES space mission, built by the Italian Limadou collaboration. Results of the test beams held at the Beam Test Facility of the INFN National Laboratory of Frascati, for electrons, and at the Proton Cyclotron of Trento, for protons, will be presented.
\end{abstract}

The European Physical Society Conference on High Energy Physics

5-12 July, 2017

Venice

${ }^{*}$ Speaker. 


\section{The High-Energy Particle Detector}

CSES is a Chinese space mission with different goals [1]:

- the measurement of signals from electromagnetic emission in ionosphere;

- the measurement of background magnetic field in space;

- the cosmic ray measurements in an energy range up to few hundreds of MeV.

The satellite will be placed in a 97,4 $4^{\circ}$ inclination Sun-synchronous circular orbit, at an altitude of $\sim 500 \mathrm{~km}$. The High-Energy Particle Detector (HEPD) is one of the payloads of CSES. It is built by the Italian collaboration Limadou. It is developed to study protons and electrons in the energy range [3-200] $\mathrm{MeV}$ [2].

HEPD is composed by different instruments [3]:

- a tracker, made of two planes of double-side silicon micro-strip sensors;

- the trigger plane, which is divided into 6 segments;

- a calorimeter, composed by 16 plastic scintillators with dimensions $(15 \times 15 \times 1) \mathrm{cm}^{3}$ and a layer of 9 LYSO cubes, for a resulting plane of dimension $(15 \times 15 \times 4) \mathrm{cm}^{3}$;

- a scintillator veto system, $5 \mathrm{~mm}$ thick, at sides and at the bottom of the instrument.

\section{Test beams}

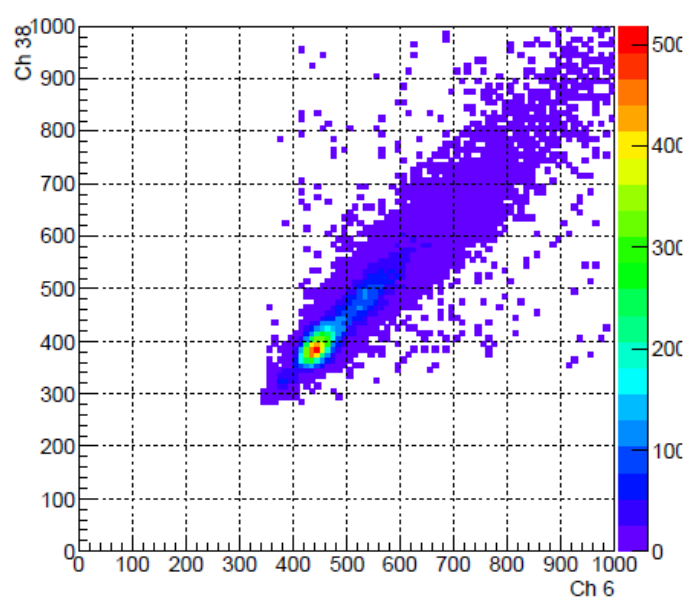

Figure 1: Counts on the 2 PMTs of the first paddle of the trigger plane induced by an electron beam with $30 \mathrm{MeV}$.

In May 2017, the detector was tested at the Beam Test Facility (BTF) of the 'Laboratori Nazionali di Frascati' of INFN with an electron/positron beam with energy between 30 and 120 $\mathrm{MeV}$ [5]. Each paddle in the trigger plane is read by 2 PMTs; the raw signal on one paddle of the trigger plane for an electron beam with energy equal to $30 \mathrm{MeV}$ is reported in Fig. 1. 


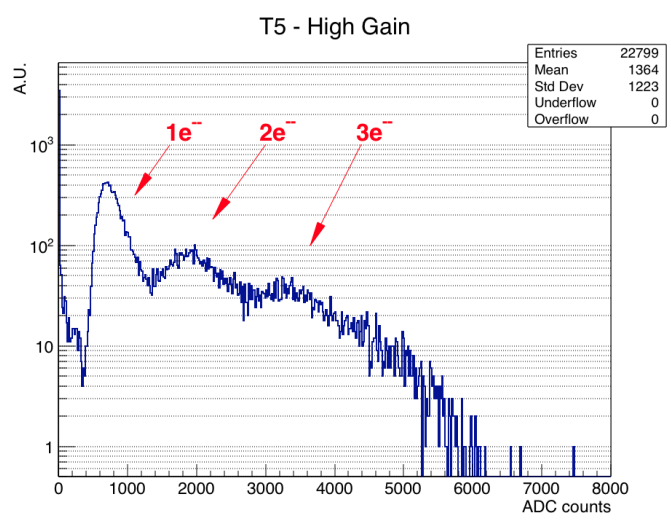

Figure 2: Energy released by an electron beam of $\mathrm{E}=30 \mathrm{MeV}$ on one paddle of the trigger plane.

In Fig. 2 the numbers of counts on the trigger paddle hit by an electron beam of $30 \mathrm{MeV}$ has been showed. It can be clearly seen the signal induced by 1, 2, and 3 contemporary particles. In Fig. 3 is reported the number of hit planes with two electron beams with different energies. The Blue plot is referred to a beam with energy equal to $30 \mathrm{MeV}$; the green plot is refererred to an electron beam with energy equal to $45 \mathrm{MeV}$. At this energy electrons pass through the 16 planes stopping in LYSO cristals. This is consistent with the energy loss of minimum ionizing particles in plastic scintillator $(\sim 2 \mathrm{MeV} / \mathrm{cm})$.

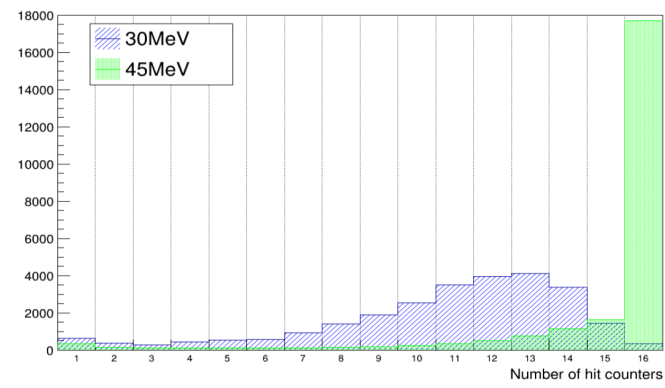

Figure 3: Number of hit planes for electron beams of $30 \mathrm{MeV}$ (Blue) and $45 \mathrm{MeV}$ (Green).

In October 2017, the detector was tested at the Proton Cyclotron of Trento with proton beams with energy included into the range [70-200] MeV. In Fig. 4 the number of hit planes for proton beams with different energies is reported. Differently from electrons, here the plane can be clearly see where protons lose all the energy.

This is also reported in Fig. 5, where the average of the total counts obtained in the upper calorimeter is reported as a function of the beam energy. The average number of counts for electron beams is constant, as for energies greater than $45 \mathrm{MeV}$ electrons cross all the upper calorimeter. For proton beams, instead, the average number of counts increases with the energy of the incident particle. 


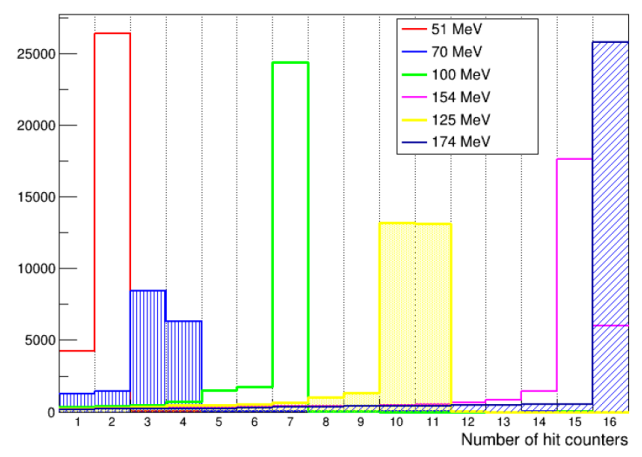

Figure 4: Number of hit planes for proton beams with different energies.

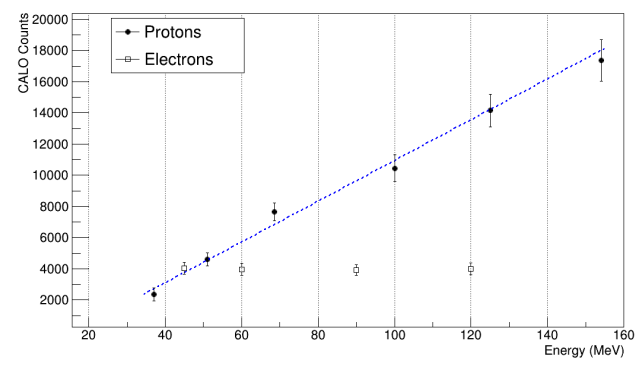

Figure 5: Average counts on the upper calorimeter for electrons (white squares) and protons (black dots).

\section{Conclusions and Acknowledgements}

We would like to thank the Beam Test Facility at 'Laboratori Nazionali di Frascati' and the Proton Cyclotron of Trento for the possibility to test the detector. In this paper some results obtained for HEPD at the test beams are reported. More data are under study.

\section{References}

[1] Wang L. et al., Earthq Sci (2015) 28 4, 303

[2] Picozza P. et al., Proceedings of the 35th ICRC 2017, Busan, South Corea

[3] Sparvoli et al., Proceedings of the Living Planet Symposium 2016, Prague, Czech Republic

[4] Panico B. et al., Proceedings of the 25th ECRS 2016 Turin, Italy, arXiv:1701.08827

[5] Ghigo A. et al., (2003) Nucl.Instrum.Meth. A 515, 524 